\title{
Myths and truths on dietary supplements and nutraceuticals for musculoskeletal health: a scoping review
}

\section{BACKGROUND}

The market of supplements and nutraceuticals is growing, especially with the pe nutrents might contribute to the reduction of muscl. mass and cognitive functions in increaris in supplements, in order to identify, according to an evidence-based approach, which of elderly: bone, skeletal muscle and nervous tissues.

\section{METHODS}

Our group performed this scoping review with the steps: (1) configuration of the Italian study group on Healthy Aging by Nutraceuticals and Dietary Supplements (HANDS); (2) identification of a list of micronutrients selected from the "EU Register of nutrition and health claims made on foods" [6] that have a health relationship with musculoskeletal or cognitive and neurological functions; (3) planning a research on PubMed, using as MeSH (Medical Subject Headings) including specific terms for the selected micronutrient, and adding for each of them (in the PubMed Search Builder) the following terms: "bone", "skeletal muscle", and "central nervous system"/"brain"/"cognitive function"; (4) searching for all the studies published in the last 10 years (up to August 2015), including only ones in English and performed on elderly people; (5) after methodological quality assessment, we identified, for each micronutrient in each area, the most relevant positive study, according to EBM pyramid: metanalysis, systematic reviews, randomized controlled trials (RCTs), cohort studies, case control studies, case series, and case reports. In case of some studies had the same level of methodological quality, we considered the most recent one. For each area involved, if international guidelines concerning the effectiveness of a micronutrient existed, these were considered as reference; (6) selection of micronutrients effective in improving health status in one or more of the three areas impaired in elderly (bone, skeletal muscle, and CNS) according to relevant studies identified; (7) identification of the effective daily doses of micronutrients, established according to the selected studies; (8) identification of adequate daily doses according to Recommended Daily Allowances (RDAs) and to tolerable upper intake levels (UL) [7]: (9) list of the resulting micronutrients, with the effective and safe daily doses, that improve health status in one or more of the three areas usually impaired in elderly.

\section{RESULTS}

From the 65 micronutrients listed on "EU Register of nutrition and health claims made on foods" [6] we identified a list of 39 micronutrients having a healthy association with musculoskeletal or cognitive and neurological functions. We reported in Table 2 relevant positive studies selected according to evidence-based approach for each micronutrient, and we identified: 1 international society guideline recommendations, 1 systematic review, 7 RCTs, and 3 prospective cohort studies. In Table 3, we described the effective daily doses of the 16 selected micronutrients that have been shown to improve health status in elderly people. Table 4 shows the effective and safe daily doses of the 16 selected micronutrients that improve health status in one or more of the three areas usually impaired in elderly people.

\section{CONCLUSIONS}

Our scoping review shows that selected micronutrients in appropriate doses might have an ancillary role in musculoskeletal health and cognitive functions in older people. Therefore, an adequate nutrition or intake of dietary supplements might have a key role in order to have a good health status.
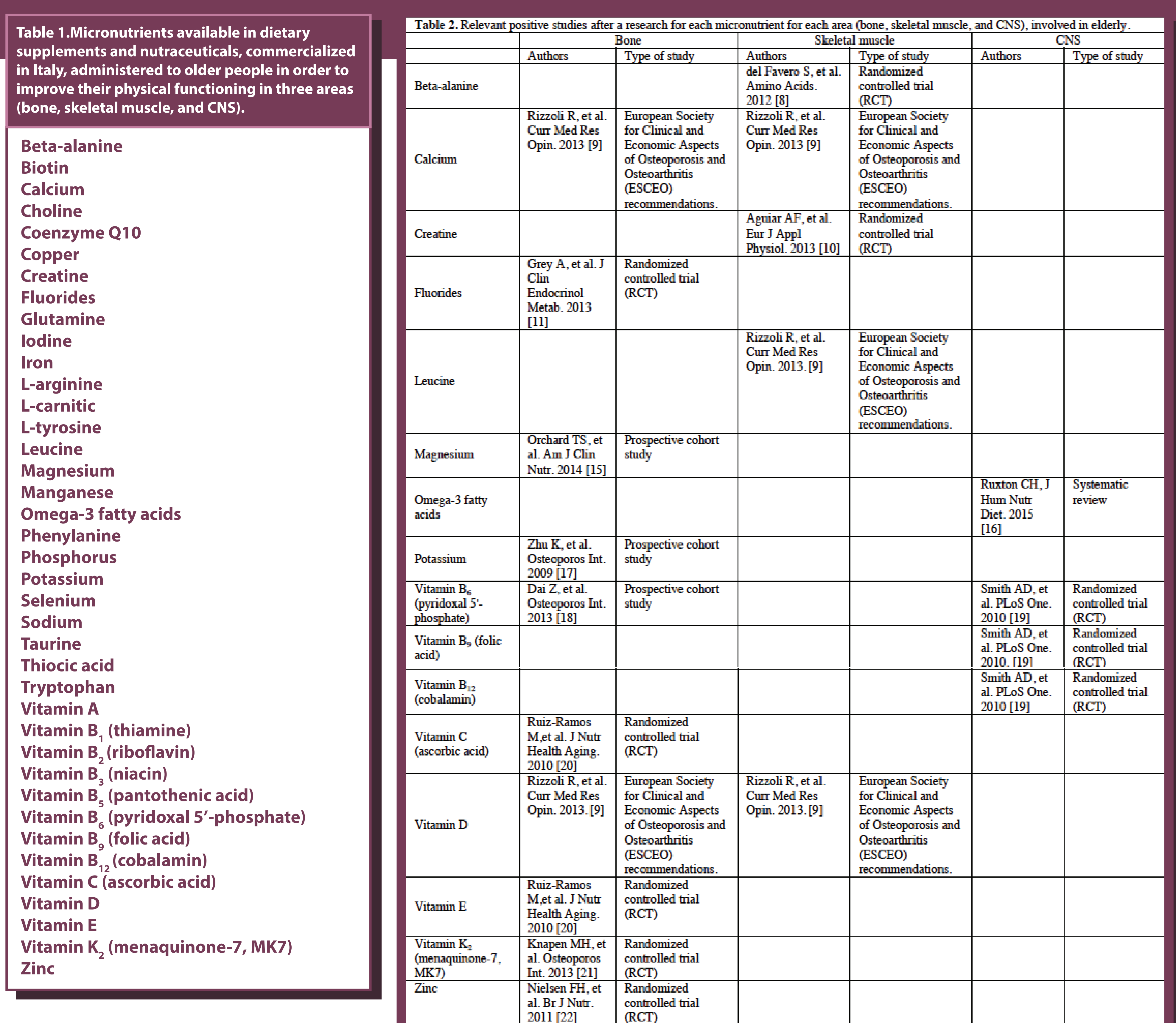

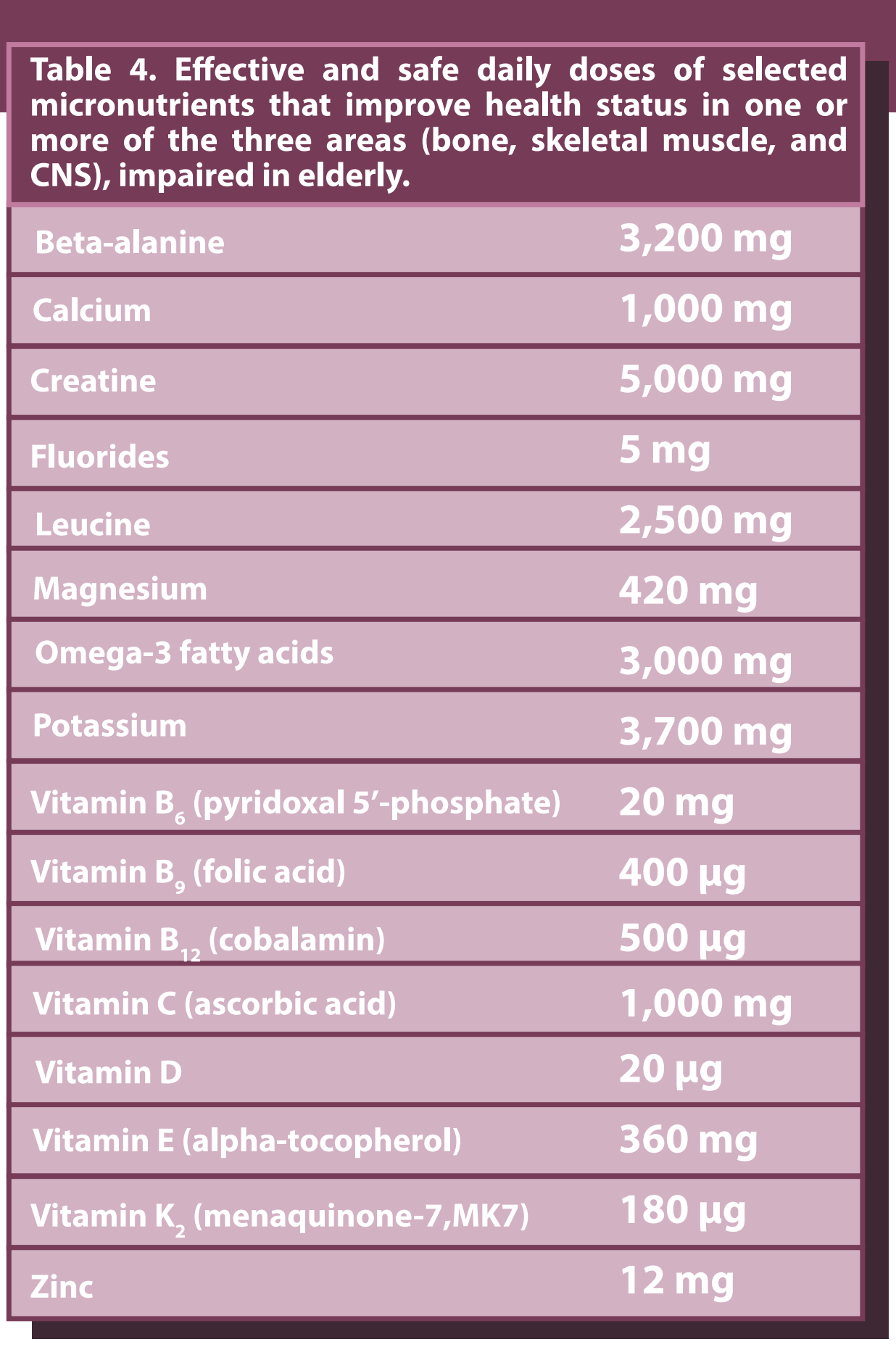

\section{REFERENCES \\ RT:}
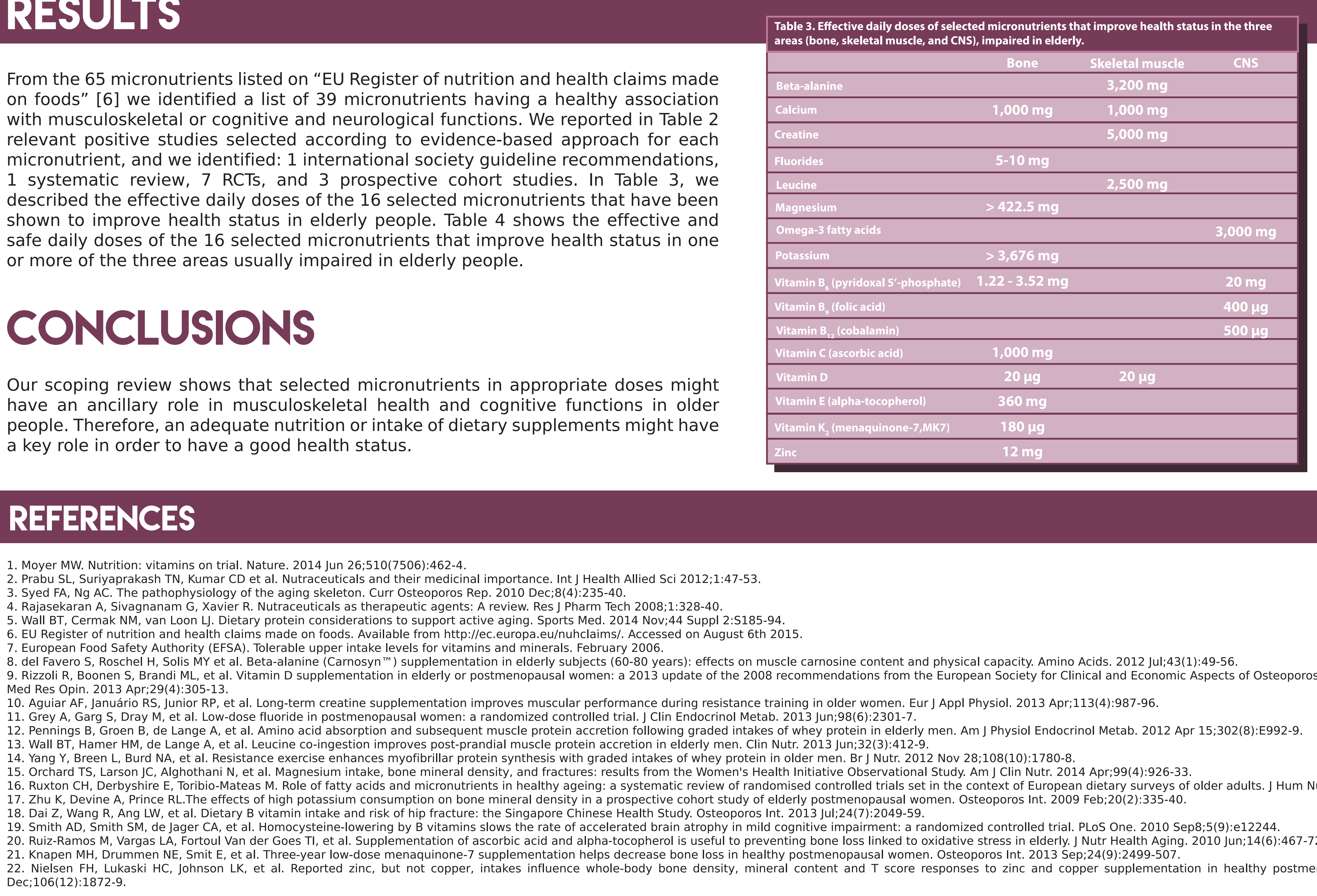

IOF

CAPTURE the

FRACTURE

Gold 